\title{
Tracheal amyloidosis
}

\author{
Ruwan Amaratunga MD FRCPC, Krishna Sharma MD FRCPC, Kayvan Amjadi MD FRCPC
}

\section{CASE PRESENTATION}

A 62-year-old woman was referred to respirology for a tracheal mass on computed tomography scan. She experienced cough six months before presentation; however, at the time of assessment, there was no fever, weight loss, cough, hemoptysis or dysphagia.

Her medical history was unremarkable. She worked as a housekeeper and denied any relevant occupational exposures. She was a 20 pack-year smoker and had quit one year previously. Physical examination was unremarkable, with no evidence of cachexia, hypoxemia, clubbing, stridor, dysphonia, heart failure, rash or focal neurological findings.

Computed tomography imaging revealed nodular thickening of the trachea and focal bronchiectasis in the lingula and right middle lobe (Figure 1). There was no evidence of parenchymal lung disease. Spirometry was normal, with no evidence of central or small airway obstruction.

Bronchoscopy revealed sessile masses along the cartilaginous walls of the trachea and mainstem bronchi (Figure 2). The lesions were gelatinous and, while they appeared friable, did not bleed. Biopsy revealed fibrillar material that stained positive for Congo red and demonstrated apple-green birefringence under polarized light consistent with amyloid.

Assessment by hematology ruled out systemic or other organspecific amyloidosis. Over the past 1.5 years of follow-up, the airway lesions have been intermittently debulked by interventional pulmonology.

\section{KEY LEARNING POINTS}

- Amyloidosis can be primary or secondary (ie, multiple myeloma) (1).

- Most cases are sporadic and can be either systemic or organ specific. However, a minority is heredofamilial in nature and these cases tend to have systemic involvement. Any part of the respiratory tract can be involved (2).

- Tracheobronchial amyloidosis is usually not associated with parenchymal (interstitial) or systemic involvement and, therefore, has a better prognosis (2).

- In proximal airway disease, spirometry is only affected once airway diameter is $<8 \mathrm{~mm}$ (3).

- If the distal airways are involved, spirometry often reveals airflow obstruction (4).

- Lesions should only be debulked once they cause symptoms (5).

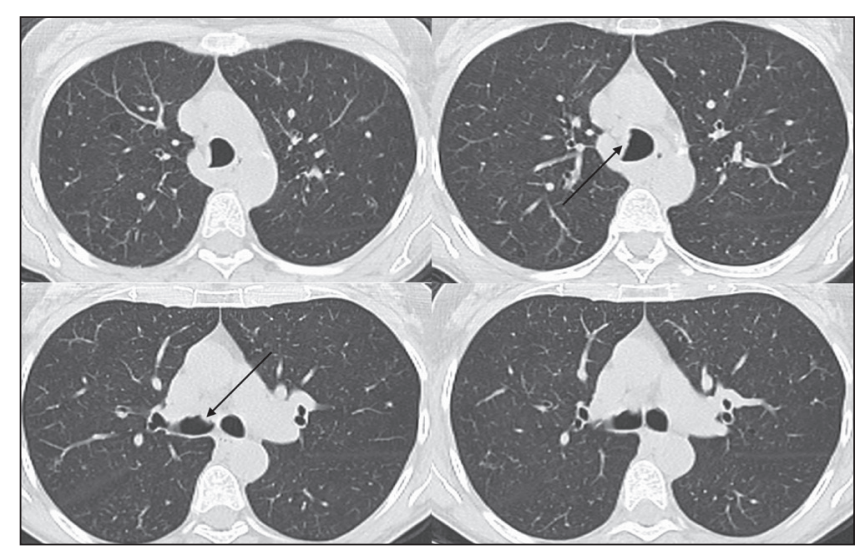

Figure 1) Computed tomography thorax images showing endoluminal lesions involving the distal trachea and right main stem bronchus (arrows)
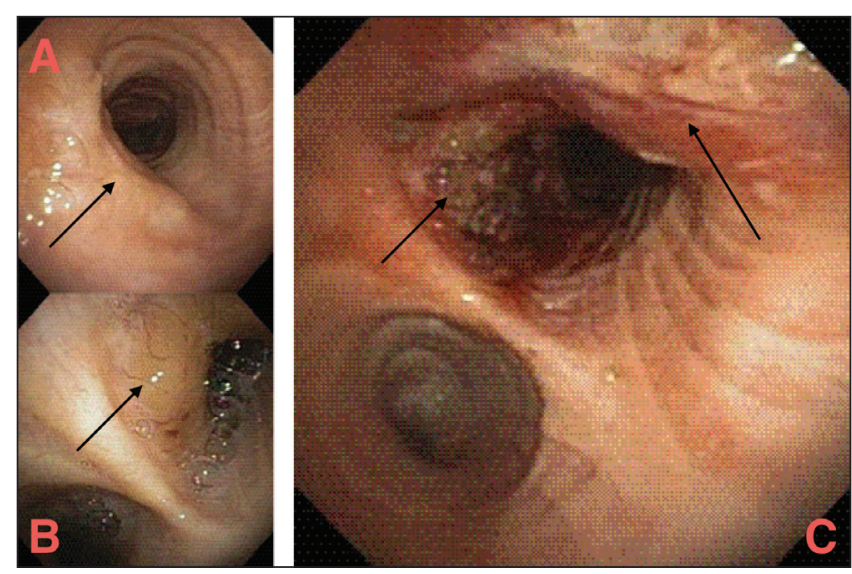

Figure 2) Amyloid plaques before manipulation in the distal trachea (A) and proximal right mainstem bronchus (B) (arrows). The same right mainstem lesion is seen following gold probe cautery (C) (arrows)

\section{REFERENCES}

1. Howard ME1, Ireton J, Daniels F, et al. Pulmonary presentations of amyloidosis. Respirology 2001;6:61-4.

2. O'Regan A1, Fenlon HM, Beamis JF Jr, Steele MP, Skinner M, Berk JL.Tracheobronchial amyloidosis. Medicine 2000;79:69-79.

3. Pribitkin E1, Friedman O, O'Hara B, et al. Amyloidosis of the upper aerodigestive tract. Laryngoscope 2003;113:2095-101.

4. Miller RD, Hyatt RE. Obstructing lesions of the larynx and trachea. Mayo Clin Proc; 44:145-61.

5. Hawkins PN. The diagnosis, natural history and treatment of amyloidosis. The Goulstonian Lecture 1995. J Roy Coll Phys Lond 1997;31:552-60

The 'Images in Respiratory Medicine' section of the Canadian Respiratory Journal aims to highlight the importance of visual interpretation, whether physiological, radiological, bronchoscopic, surgical/thorascopic or histological, in the diagnosis of chest diseases. Submissions should exemplify a classic, particularly dramatic or intriguing presentation of a disease while offering an important educational message to the reader (insightful diagnostic pearls or differential diagnosis, etc). This section is not intended to be a vehicle for publication of case reports (see the Clinical-Pathologic Conferences for case-based leaning series).

Department of Medicine, Division of Respirology, The Ottawa Hospital, Ottawa, Ontario

Corresponence: Dr Ruwan Amaratunga, 981 Torovin Private, Ottawa, Ontario K1B 0A6. Telephone 613-680-4590,

e-mail ramaratunga@gmail.com 


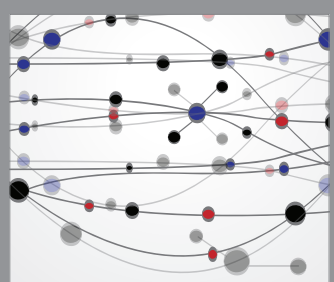

The Scientific World Journal
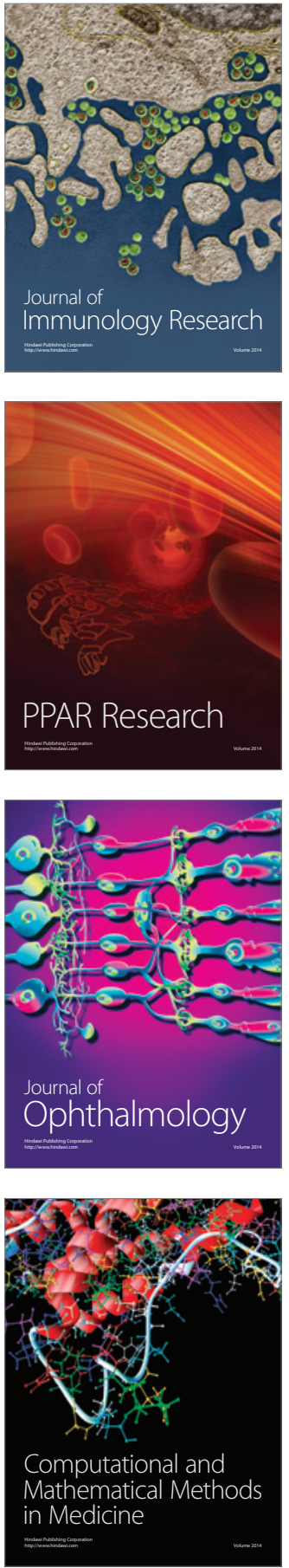

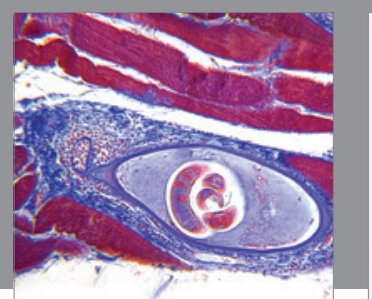

Gastroenterology Research and Practice

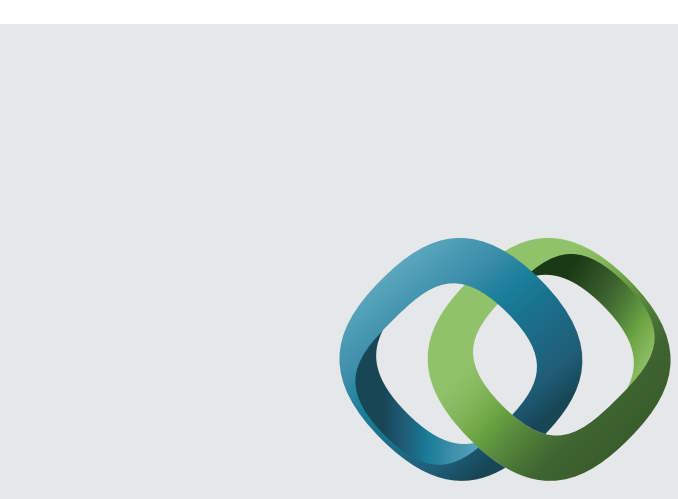

\section{Hindawi}

Submit your manuscripts at

http://www.hindawi.com
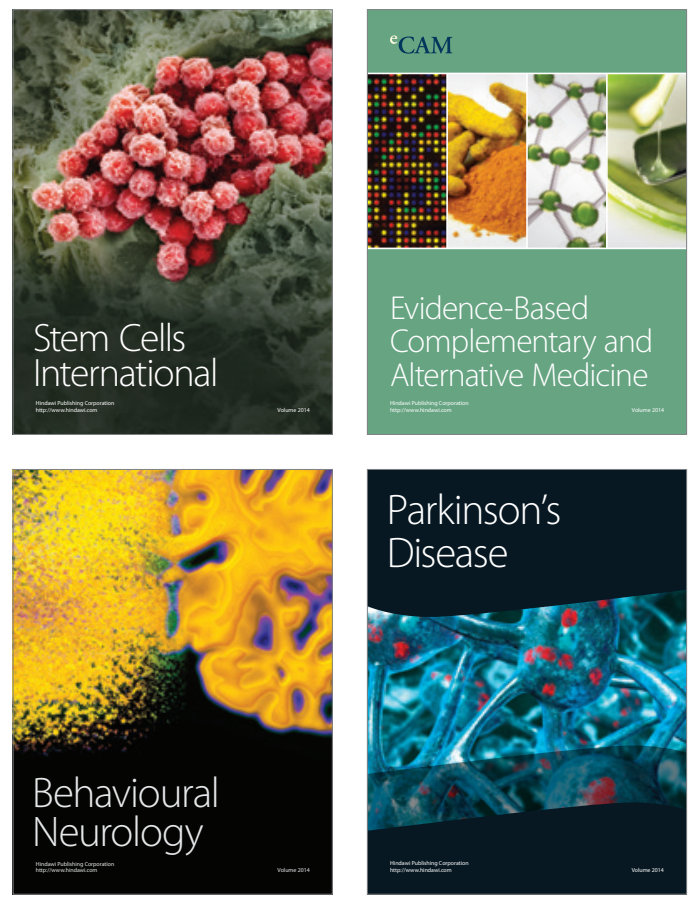
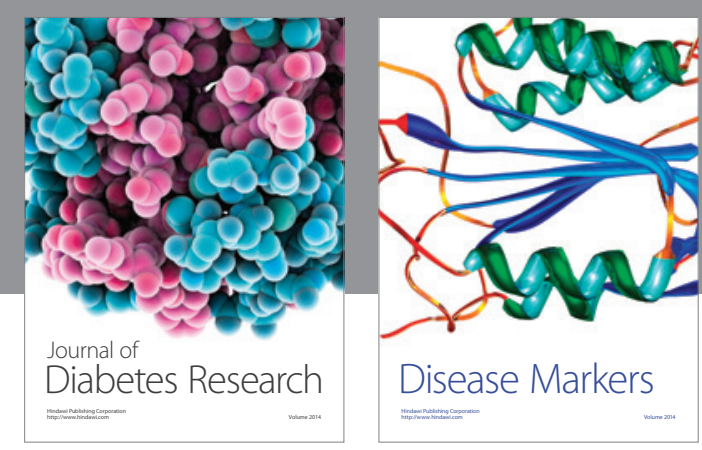

Disease Markers
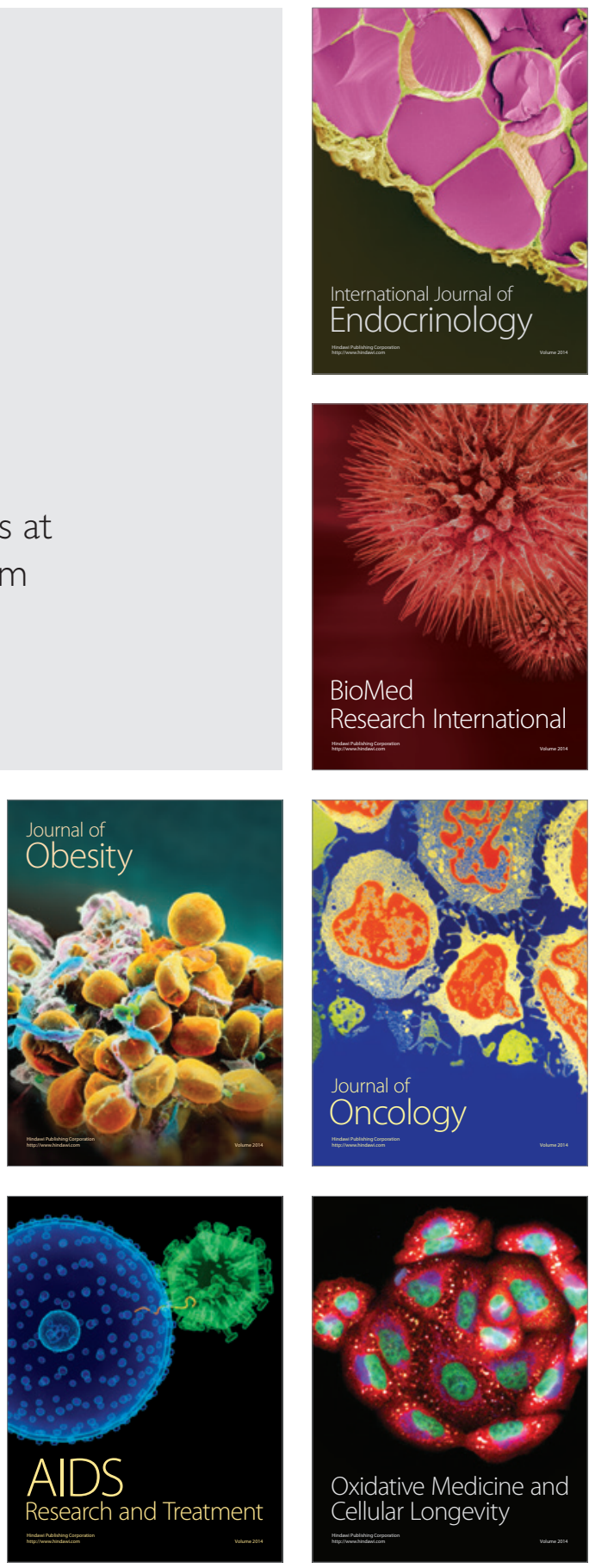